\title{
Giant higher harmonic generation in mesoscopic metal wires and rings interrupted by tunnel junctions
}

\author{
A. van Oudenaarden, ${ }^{*}$ Yu. V. Nazarov, and J. E. Mooij \\ Department of Applied Physics and Delft Institute of Micro-electronics and Submicron-technology (DIMES), Delft University of \\ Technology, P.O. Box 5046, 2600 GA Delft, The Netherlands
}

(Received 19 December 1997)

\begin{abstract}
Higher harmonic generation in mesoscopic metal wires and loops interrupted by tunnel junctions is explored experimentally. When the sample is biased with a sinusoidal varying current, we observe giant higher harmonics in the conductance fluctuations and the Aharonov-Bohm oscillations. We demonstrate that the harmonic content is directly related to the probability for an electron to return to the barrier after tunneling. The full set of experimental data is in quantitative agreement with theoretical predictions. [S0163-1829(98)01915-8]
\end{abstract}

The classical model of conductivity, which completely omits the wave nature of electrons, needs revision when the size of a metal sample is of order of micrometers or less. In these mesoscopic samples electrons can traverse the metal phase coherently, which introduces significant corrections to the classical model due to quantum interference effects of electron waves. The experimental observations of weak localization, Aharonov-Bohm effect, and universal conductance fluctuations have clearly demonstrated the important role of quantum interference in transport properties. ${ }^{1} \mathrm{An}-$ other important consequence of quantum interference, which is less well understood, is the nonlinear nature of the conductance. ${ }^{2}$ A powerful method to characterize this nonlinearity experimentally is to study higher harmonic generation. The current through the sample is driven at a fundamental frequency $\nu$ and the voltage is detected at the harmonics $N \nu$, where $N=2,3,4, \ldots$. Earlier experiments on metal samples $^{3}$ and ballistic GaAs structures ${ }^{4}$ showed the presence of higher harmonics, but the results were not understood completely. An experimental disadvantage of these systems is the fact that it is very difficult to apply an abrupt voltage drop across the sample. The voltage drop from one lead to the other is determined by the microscopic arrangement of impurities. This problem is circumvented when the wire is interrupted by a tunnel barrier. ${ }^{5}$ In this system the voltage drops almost completely over the tunnel barrier, since the resistance of the tunnel junction is much larger than the resistance of the metal leads. The ability to apply an abrupt voltage drop is a necessary condition for a systematic study of higher harmonic generation. In this paper we experimentally explore the generation of higher harmonics in mesoscopic wires and rings. We study both the harmonic content of aperiodic conductance fluctuations and periodic Aharonov-Bohm oscillations and demonstrate the significantly different nonlinear response. The experiments are quantitatively described within a theoretical framework.

The higher harmonic generation has been studied in two types of samples: an Aharonov-Bohm ring interrupted by two small tunnel junctions and a metal wire interrupted by one tunnel junction. The mask of the samples was defined by electron beam lithography in a double layer PMMA (polymethylmethacrylate) resist. Using a shadow evaporation technique the overlap $\mathrm{Al}-\mathrm{Al}_{2} \mathrm{O}_{3}-\mathrm{Al}$ tunnel junctions were formed. The width of the arms of the aluminum ring is 60 $\mathrm{nm}$ and the circumference of the loop is $3.2 \mu \mathrm{m}$. The total thickness of the wires is $65 \mathrm{~nm}$. By determining the overlap areas of the two junctions we conclude that the conductances of the two junctions do not differ by more than $10 \%$. This is confirmed by critical current measurements in the superconducting state in which the device operates as a SQUID. The tunnel conductance $G_{T}$ of the two junctions in parallel is 125 $\mu \mathrm{S}$. The wire interrupted by one tunnel junction is fabricated in the same way as the ring. The width of the left and right parts of the wire are 200 and $400 \mathrm{~nm}$, respectively. The thickness of the wire is $65 \mathrm{~nm}$. The tunnel conductance of the single junction is $57 \mu \mathrm{S}$. Both the Aharonov-Bohm loop and the single wire are capacitively coupled on-chip to a low impedance platinum shunt resistor to create a well-defined electromagnetic environment.

Measurements were performed in a dilution refrigerator at a base temperature of $20 \mathrm{mK}$. Electrical leads were carefully filtered by microwave copper powder filters and $R C$ filters at base temperature. At room temperature the leads were additionally filtered by $\Pi$ filters. The sample was mounted in two microwave-tight copper boxes at base temperature and an additional shield at $1 \mathrm{~K}$. A sinusoidal current was injected into the sample at $\nu=17 \mathrm{~Hz}$. The voltage response was detected using a lock-in amplifier synchronized to one of the higher harmonics $N \nu$.

The samples were biased with a current $I=\bar{I}$ $+I_{m} \cos (2 \pi \nu t)$, where $\bar{I}$ is the dc component of the bias current and $I_{m}$ the amplitude of the time-varying component. Examples of a voltage response detected at the harmonic $N$ for the Aharonov-Bohm oscillations and the aperiodic voltage fluctuations are plotted in Figs. 1(a) and Fig. 1(b), respectively. The dc component $\bar{I}=50 \mathrm{nA}$ results in an average voltage drop $\bar{V}=\bar{I} / G_{T}=0.4 \mathrm{mV}$ and the ac component $I_{m}=2.5 \mathrm{nA}$ results in a time-varying voltage across the sample with amplitude $V_{m}=I_{m} / G_{T}=20 \mu \mathrm{V}$. The variance of the conductance $\left\langle\delta G^{2}\right\rangle$ is directly proportional to $\bar{V}$, which was explored in detail in Ref. 5. The voltage $V_{N}$ is defined as the voltage detected at frequency $N \nu$. In Figs. 1(a) and 1(b) the voltages $V_{1}, V_{2}$, and $V_{3}$ are plotted versus magnetic field $B$ for $V_{m}=20 \mu \mathrm{V}$ and sample temperature $T=$ $20 \mathrm{mK}$. Note that magnetic fields are large enough to drive the aluminum into the normal state. Figure 1(a) shows clear 


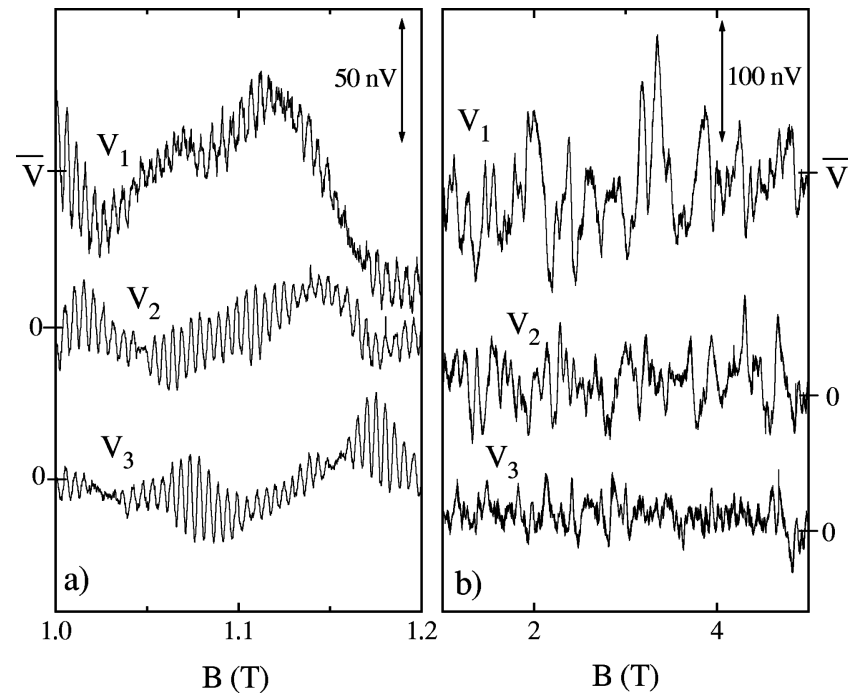

FIG. 1. Aharonov-Bohm voltage oscillations (a) and aperiodic voltage fluctuations (b) vs magnetic field $B$ detected at the fundamental frequency $V_{1}$, the second harmonic $V_{2}$, and third harmonic $V_{3}\left(V_{m}=20 \mu \mathrm{V}, T=20 \mathrm{mK}\right)$. The curves are shifted with respect to the $y$ axis for clarity.

Aharonov-Bohm oscillations with a period of $5 \mathrm{mT}$, which corresponds to adding one flux quantum $h / e$ to the ring. The slowly varying background superposed on the AharonovBohm oscillations is due to the fact that magnetic flux also penetrates the arms of the loop, which leads to aperiodic conductance fluctuations. When these fluctuations are filtered out digitally using Fourier analysis the voltage oscillations due to the Aharonov-Bohm effect are separated from the aperiodic voltage fluctuations. In this way the variance of the voltage oscillations $\left\langle V_{N}^{2}\right\rangle$, detected at the $N$ th harmonic, is calculated. Analyzing the Aharonov-Bohm oscillations in the range between 1.0 and $1.5 \mathrm{~T}$ (100 Aharonov-Bohm periods) results in $\left\langle\delta V_{1}^{2}\right\rangle=43(\mathrm{nV})^{2},\left\langle\delta V_{2}^{2}\right\rangle=39(\mathrm{nV})^{2}$, and $\left\langle\delta V_{3}^{2}\right\rangle$ $=44(\mathrm{nV})^{2}$. These values are accurate to within $20 \%$. Surprisingly the amplitude of the Aharonov-Bohm oscillations detected at the second and third harmonic are approximately the same as the Aharonov-Bohm oscillations detected at the driving frequency $\nu$. This giant higher harmonic generation for Aharonov-Bohm oscillations was not observed in earlier experiments. ${ }^{3,4}$ An example of higher harmonic generation for the aperiodic voltage fluctuations is shown in Fig. 1(b). The Aharonov-Bohm oscillations are digitally filtered out using Fourier analysis. The variance of the voltage fluctuations is computed from a set of 4000 data points measured from 1.0 to $5.0 \mathrm{~T}$. In contrast to the Aharonov-Bohm oscillations the aperiodic fluctuations decrease for increasing $N:\left\langle\delta V_{1}^{2}\right\rangle$ $=1260(\mathrm{nV})^{2},\left\langle\delta V_{2}^{2}\right\rangle=411(\mathrm{nV})^{2}$, and $\left\langle\delta V_{3}^{2}\right\rangle=138(\mathrm{nV})^{2}$.

The Aharonov-Bohm oscillations and the conductance fluctuations in a system with tunnel junctions are due to interference of electron and hole trajectories as depicted in Fig.

2. At a finite dc bias voltage $\bar{V}$ electric transport occurs when an electron tunnels from an occupied state in the left part to an empty state in the right part of the sample [Fig. 2(a)]. At the moment of tunneling an electron-hole pair is created, which starts to diffuse through the sample. In the wire geometry [Fig. 2(b)], only those electron-hole trajectories that recombine at the tunnel barrier coherently contribute to the

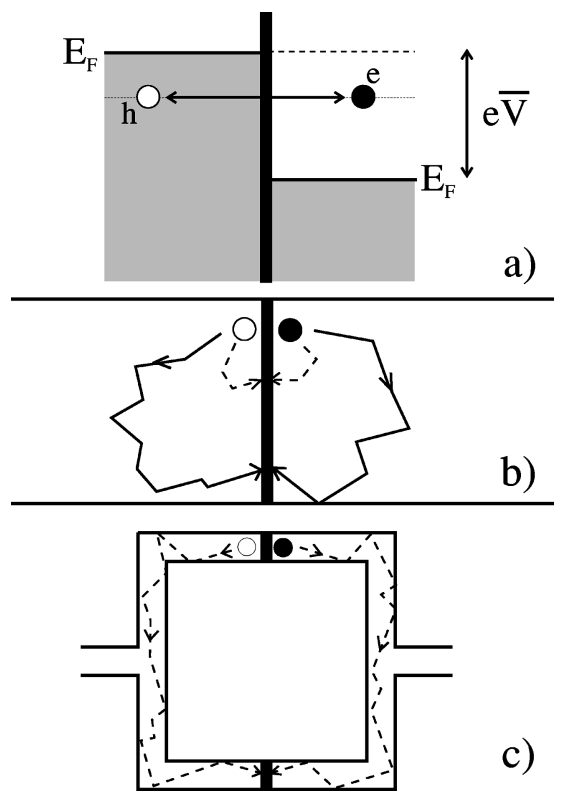

FIG. 2. (a) An electron tunnels into an empty state above the Fermi energy $E_{F}$ in the right part-leaving a hole in the left part. (b) Two possible electron-hole trajectories that contribute to the voltage fluctuations. (c) Electron-hole trajectory that contributes to the Aharonov-Bohm oscillations.

conductance fluctuations $(\mathrm{CF})$. For one-dimensional diffusion the classical return probability for the electron or hole is

$$
P_{\mathrm{cl}}^{\mathrm{CF}}(t) \approx \frac{1}{S \sqrt{\pi t D}},
$$

where $D$ is the diffusion coefficient and $S$ the wire cross section. Most of the electron-hole pairs interfere within a time $t$ close to zero. However, in the Aharonov-Bohm ring [Fig. 2(c)] the return probability is maximum at $t$ $=L^{2} /(2 D)$, where $L$ is the distance between the tunnel barriers, and the return probability is exponentially damped for small $t$. Only those electron-hole trajectories that are created at one junction and recombine at the other contribute to the Aharonov-Bohm (AB) conductance. The return probability is given by ${ }^{6}$

$$
P_{\mathrm{cl}}^{\mathrm{AB}}(t) \approx \frac{1}{S \sqrt{\pi t D}} e^{-L^{2} /(4 D t)} .
$$

The functional behavior of $P_{\mathrm{cl}}^{\mathrm{AB}}(t)$ and $P_{\mathrm{cl}}^{\mathrm{CF}}(t)$ are depicted in the insets of Figs. 4(a) and 4(b), respectively. The significant difference between $P_{\mathrm{cl}}^{\mathrm{AB}}$ and $P_{\mathrm{cl}}^{\mathrm{CF}}$ results in a different higher harmonic response as was already demonstrated experimentally in Figs. 1(a) and 1(b). The variance of the voltage analyzed at harmonic $N$ is given by ${ }^{6}$

$$
\left\langle\delta V_{N}^{2}\right\rangle=A \int_{0}^{\infty} d t P_{\mathrm{cl}}^{L}(t) P_{\mathrm{cl}}^{R}(t) e^{-2 t / \tau_{\varphi}} J_{N}^{2}\left(\frac{e}{\hbar} V_{m} t\right),
$$

where $J_{N}^{2}\left[(e / \hbar) V_{m} t\right]$ is the square of the $N$ th-order Bessel function of the first kind. The dephasing time $\tau_{\varphi}$ describes the loss of coherence due to inelastic processes. The factor 2 arises because both the electron and hole contribute a factor $e^{-t / \tau_{\varphi}}$ to the integral. The prefactor $A=4 \bar{V} /\left(\pi e \hbar \nu_{L} \nu_{R}\right)$ captures all variables that are time independent. The density of 


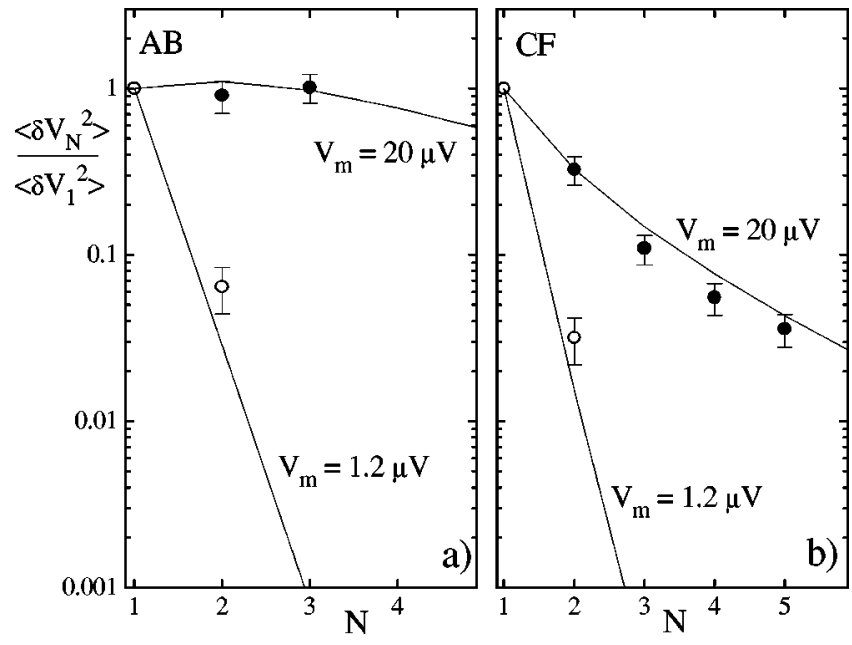

FIG. 3. Normalized variance of the Aharonov-Bohm oscillations (a) and voltage fluctuations (b) vs $N$ at large and small $V_{m}$. The full lines denote the theoretical predictions. Note that the $y$ axis is plotted on a logarithmic axis.

states in the left and right electrodes are denoted by $\nu_{L}$ and $\nu_{R}$. The angular brackets denote an average over impurity configurations that is experimentally achieved by sweeping the magnetic field.

The maximum of $J_{N}^{2}\left[(e / \hbar) V_{m} t\right]$ in Eq. (3) is located near $\tilde{t} \approx(N+1) \hbar /\left(e V_{m}\right)$. This function acts as a kind of bandpass filter for the interference time $t$. The variance $\left\langle\delta V_{N}^{2}\right\rangle$ is predominantly determined by electron-hole pairs, which interfere in a time $t \approx \widetilde{t}$. When $N$ is increased, or equivalently $V_{m}$ is decreased, $\left\langle\delta V_{N}^{2}\right\rangle$ is determined by electron-hole pairs that interfere after longer times. Because the return probability of electron-hole pairs contributing to the conductance fluctuations decreases monotonously for decreasing $t,\left\langle\delta V_{N}^{2}\right\rangle$ is always maximal for $N=1$ or $V_{m} \rightarrow \infty$, which is confirmed experimentally in Figs. 3(b) and 4(b). However, $P_{\mathrm{cl}}^{\mathrm{AB}}(t)$ has a maximum near $t \approx L^{2} /(2 D)$, which leads to a maximum $\left\langle\delta V_{N}^{2}\right\rangle$ for $N \neq 1$ and finite $V_{m}$, shown in Figs. 3(a) and 4(a) for $V_{m}=20 \mu \mathrm{V}$. For $V_{m}=1.2 \mu \mathrm{V}$, however, $\left\langle\delta V_{2}^{2}\right\rangle$ is more than one order of magnitude smaller than $\left\langle\delta V_{1}^{2}\right\rangle$ for both the Aharonov-Bohm oscillations and the voltage fluctuations. For very small $V_{m}$ the dephasing time $\tau_{\varphi}$ is smaller than $\tilde{t}$, which leads to exponentially decreasing higher harmonics. The factor $e^{-2 t / \tau_{\varphi}}$ in Eq. (3) acts as a low-pass filter for $t$.

To compare the experimental data to the theory, expressed by Eqs. (1)-(3), the dephasing time $\tau_{\varphi}$ and the dif-
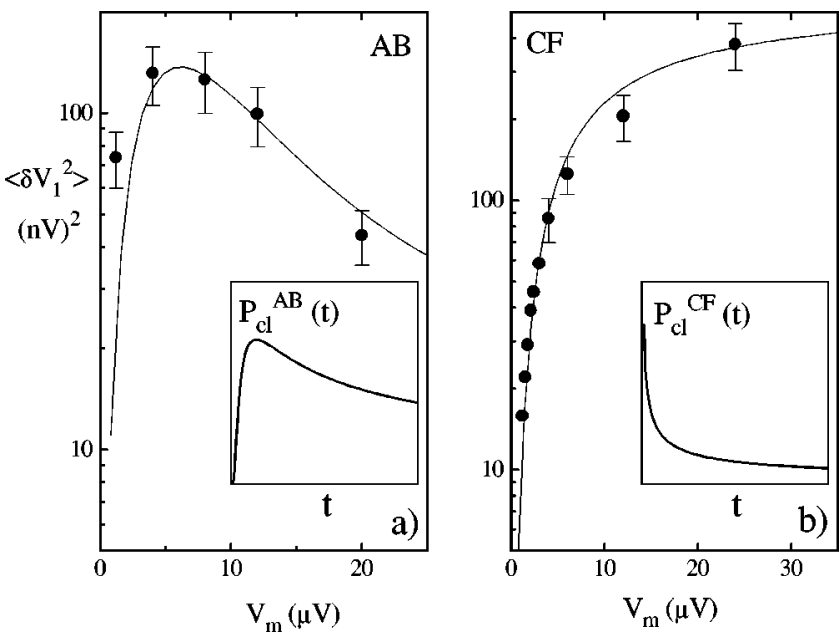

FIG. 4. The variance of the fundamental response of the Aharonov-Bohm oscillations (a) and the aperiodic fluctuations (b) vs excitation voltage $V_{m}$. The full lines denote the theoretical predictions. Note that the $y$ axis is plotted on a logarithmic axis. The functional behavior of $P_{\mathrm{cl}}^{\mathrm{AB}}(t)$ and $P_{\mathrm{cl}}^{\mathrm{CF}}(t)$ is shown in the insets.

fusion time $L^{2} / D$ have to be determined. A quantitative agreement between experiment and theory is obtained for $\tau_{\varphi}=0.23 \pm 0.05 \mathrm{~ns}$ and $L^{2} / D=0.5 \pm 0.2 \mathrm{~ns}$. The value of $\tau_{\varphi}$ is consistent with values usually found in mesoscopic metal systems. ${ }^{5,7}$ The distance between the tunnel barriers $L=1.6$ $\mu \mathrm{m}$ leads to a diffusion coefficient $D=5 \times 10^{-3} \mathrm{~m}^{2} / \mathrm{s}$, which is consistent with $D=\frac{1}{3} v_{F} \ell$, where $v_{F}=2 \times 10^{-6} \mathrm{~m} / \mathrm{s}$ (Ref. 8 ) is the free electron Fermi velocity in aluminum and $\ell$ the mean free path, which is approximately $10 \mathrm{~nm}$. The theoretical predictions are denoted by the full lines in Figs. 3 and 4.

In summary, we have experimentally explored the generation of higher harmonics in Aharonov-Bohm and wire geometries. We demonstrate the direct relation between the higher harmonic generation and the classical return probability, which is significantly different for the voltage fluctuations and the Aharonov-Bohm oscillations. The full set of experimental data is consistently and quantitatively described by a theoretical model with two parameters: the phase breaking time $\tau_{\varphi}$ and the diffusion coefficient.

We want to thank M. H. Devoret for valuable discussions. The Delft Institute of Micro-electronics and Submicrontechnology (DIMES) is acknowledged for the help with the fabrication. This work was financially supported by the Dutch Foundation for Fundamental Research on Matter (FOM).
*Present address: Dept. of Chemistry, Stanford University, Stanford, CA 94305-5080.

${ }^{1}$ S. Washburn and R. A. Webb, Rep. Prog. Phys. 55, 1311 (1992).

${ }^{2}$ B. L. Al'thshuler and D. E. Khmel'nitskii, Pis'ma Zh. Eksp. Teor. Fiz. 42, 291 (1985) [ JETP Lett. 42, 359 (1986)]; A. I. Larkin and D. E. Khmel'nitskii, Zh. Eksp. Teor. Fiz. 91, 1815 (1986) [Sov. Phys. JETP 64, 1075 (1986)]; A. I. Larkin and K. A. Matveev, Zh. Eksp. Teor. Fiz. 93, 1030 (1987) [Sov. Phys. JETP 66, 580 (1987)]; T. K. Ng, Phys. Rev. Lett. 68, 1018 (1992); A. Levy Yeyati, Phys. Rev. B 45, 14189 (1992).

${ }^{3}$ R. A. Webb, S. Washburn, and C. P. Umbach, Phys. Rev. B 37, 8455 (1988).
${ }^{4}$ P. G. N. de Vegvar, G. Timp, P. M. Mankiewich, J. E. Cunningham, R. Behringer, and R. E. Howard, Phys. Rev. B 38, 4326 (1988).

${ }^{5}$ A. van Oudenaarden, M. H. Devoret, E. H. Visscher, Yu. V. Nazarov, and J. E. Mooij, Phys. Rev. Lett. 78, 3539 (1997).

${ }^{6}$ Yu. V. Nazarov, Zh. Eksp. Teor. Fiz. 98, 306 (1990) [Sov. Phys. JETP 71, 171 (1990)]; Yu. V. Nazarov, Phys. Rev. B 47, 2768 (1993).

${ }^{7}$ P. Mohanty, E. M. Q. Jariwala, and R. A. Webb, Phys. Rev. Lett. 78, 3366 (1997).

${ }^{8}$ C. Kittel, in Introduction to Solid State Physics (John Wiley \& Sons, Inc., New York, 1986). 